\title{
IONIZED GAS KINEMATICS AT HIGH RESOLUTION. IV. STAR FORMATION AND A ROTATING CORE IN THE MEDUSA (NGC 4194)
}

\author{
Sara C. Beck ${ }^{1,6}$, John Lacy $^{2,6}$, Jean Turner $^{3}$, Thomas Greathouse $^{4,6}$, and Susan NefF ${ }^{5}$ \\ ${ }^{1}$ School of Physics and Astronomy, Tel Aviv University, Ramat Aviv 69978, Israel; becksarac@ gmail.com \\ ${ }^{2}$ Department of Astronomy, University of Texas at Austin, Austin, TX 78712, USA \\ ${ }^{3}$ Department of Physics and Astronomy, UCLA, Los Angeles, CA 90095-1547, USA \\ ${ }^{4}$ Southwest Research Institute, San Antonio, TX 78228-0510, USA \\ ${ }^{5}$ NASA-Goddard Space Flight Center, Greenbelt, MD 20771, USA \\ Received 2014 February 4; accepted 2014 April 7; published 2014 May 6
}

\begin{abstract}
NGC 4194 is a post-merger starburst known as The Medusa for its striking tidal features. We present here a detailed study of the structure and kinematics of ionized gas in the central $0.65 \mathrm{kpc}$ of the Medusa. The data include radio continuum maps with resolution up to 0 '. $18(35 \mathrm{pc})$ and a $12.8 \mu \mathrm{m}$ [Ne II] data cube with spectral resolution $\sim 4 \mathrm{~km} \mathrm{~s}^{-1}$ : the first high-resolution, extinction-free observations of this remarkable object. The ionized gas has the kinematic signature of a core in solid-body rotation. The starburst has formed a complex of bright compact H II regions, probably excited by deeply embedded super star clusters, but none of these sources is a convincing candidate for a Galactic nucleus. The nuclei of the merger partners that created the Medusa have not yet been identified.
\end{abstract}

Key words: galaxies: individual (NGC 4194) - galaxies: kinematics and dynamics - galaxies: starburst - galaxies: star formation

Online-only material: color figures

\section{INTRODUCTION}

The most luminous starbursts appear to be caused by major mergers of galaxies (e.g., Sanders et al. 1988) and are rare because major mergers are infrequent. Minor mergers create LIRGS; slightly less luminous but much more common, and important to galaxy evolution due to their sheer numbers (Kartaltepe et al. 2010). Minor mergers, like major mergers, appear to instigate intense star formation in compact regions and create concentrated sources. But minor mergers evolve differently from major mergers, so the characteristics of star formation are likely to be different. How does star formation develop in a minor merger? How will these starbursts evolve and how do they affect their surroundings as they contribute to the newly formed galaxy? To answer those questions we need to understand the structure and kinematics of the gas ionized by the embedded stars with the highest possible spectral and spatial resolution. We consider here the case of NGC 4194, "The Medusa," a galaxy with distorted morphology and luminous star formation that appears to be the result of an unequal merger between an elliptical and a smaller spiral.

This is the fourth paper in a series on high-resolution spectroscopy of intense extragalactic star formation sources in the middle infrared (Beck et al. 2010, 2012, 2013). We use mid-infrared lines of metal ions to trace the kinematics and spatial distribution of ionized gas in Galactic $\mathrm{H}$ II regions and in starburst galaxies (Achtermann \& Lacy 1995; Zhu et al. 2008; Beck et al. 2013) because they are little affected by extinction and permit us to attain true spectral resolution, including thermal effects, much higher than is possible with any hydrogen line. The "Medusa galaxy," NGC 4194 (a.k.a. Arp 160 and Mkn 201) is a

\footnotetext{
6 Visiting Astronomer at the Infrared Telescope Facility, which is operated by the University of Hawaii under Cooperative Agreement no. NNX-08AE38A with the National Aeronautics and Space Administration, Science Mission Directorate, Planetary Astronomy Program.
}

starburst galaxy at $39 \mathrm{Mpc}$ distance $\left(1^{\prime \prime}=190 \mathrm{pc}\right)$ with striking tidal features; it has been variously classed as Magellanic, $\mathrm{Sm}$ (pec), and BCG. The central kpc of NGC 4194 is a powerful infrared source with $60 \mu \mathrm{m} / 100 \mu \mathrm{m} \approx 1$, typical of starburst heating. Spitzer Infrared Spectrograph (IRS) spectra (from the Heritage Archive) show a spectrum typical of a starburstdominated galaxy, with little if any contribution from an active galactic nucleus (AGN). NGC 4194 is believed to be a merger remnant, with the favored history being a small gas-rich spiral falling into an elliptical four times its mass (Manthey et al. 2008). The central region of NGC 4194 is the site of intense star formation: Weistrop et al. (2004) and Hancock et al. (2006) find numerous bright optical and UV knots that they identify as very young globular cluster precursors. But the structure of this central starburst has not been probed. The observations are either too low in spatial resolution (e.g., the Two Micron Sky Survey) or too affected by the deep extinction (Hattori et al. 2004) to determine the structure. Beswick et al. (2005) mapped the center of NGC 4194 at $21 \mathrm{~cm}$ with sub-arcsecond resolution and found two compact sources separated by only 0.35 , which they identify with the Galactic nucleus, and which Manthey et al. (2008) appear to tentatively accept as the nuclei of the progenitor galaxies, but the nature of these sources and their relation to the merger history have not been explored.

We report here on radio continuum maps of NGC 4194 with sub-arcsecond spatial resolution and on spectra of the $12.8 \mu \mathrm{m}$ emission line of [Ne II] with velocity resolution better than $5 \mathrm{~km} \mathrm{~s}^{-1}$. The radio maps are at short $\mathrm{cm}$ wavelengths, which are sensitive to both the thermal emission of $\mathrm{H}$ II regions and the non-thermal emission of supernova remnants. The finestructure line of $\mathrm{Ne}^{+}$at $12.8 \mu \mathrm{m}$ is usually one of the strongest mid-infrared emission lines in $\mathrm{H}$ II regions and is a preferred kinematic probe because of its low susceptibility to thermal broadening; in a $T_{e}=7500 \mathrm{~K} \mathrm{H}$ II region the FWHM from thermal effects will be $4.1 \mathrm{~km} \mathrm{~s}^{-1}$ compared to $18 \mathrm{~km} \mathrm{~s}^{-1}$ for $\mathrm{H}^{+}$. 
Table 1

Observations and Reductions

\begin{tabular}{|c|c|c|c|c|c|c|}
\hline Date & Instrument & Wavelength & Beam Size & $\Theta_{\text {las }}^{\mathrm{a}}$ & Noise & Program \\
\hline 2013 Feb 2 & TEXES & $12.8 \mu \mathrm{m}$ & 1 1.4 & n.a. & n.a. & n.a. \\
\hline 1987 Jul 6 & VLA & $2 \mathrm{~cm}$ & $0^{\prime \prime} 18 \times 0^{\prime \prime} .149$ & $33^{\prime \prime} 6$ & $0.06 \mathrm{mJy} \mathrm{bm}^{-1}$ & AE47 \\
\hline 1987 Nov 21 & VLA & $6 \mathrm{~cm}$ & $1^{\prime \prime} .4 \times 11^{\prime \prime} 13$ & $24^{\prime \prime}$ & $0.05 \mathrm{mJy} \mathrm{bm}^{-1}$ & AS286 \\
\hline 2000 Nov 20 & VLA & $6 \mathrm{~cm}$ & $0^{\prime \prime} .27 \times 0^{\prime \prime} .17$ & $8^{\prime \prime} .9$ & $0.08 \mathrm{mJy} \mathrm{bm}^{-1}$ & AN095 \\
\hline " & $"$ & $"$ & $0^{\prime \prime} .46 \times 0 . .43$ & 8.9 & $0.02 \mathrm{mJy} \mathrm{bm}^{-1}$ & , \\
\hline$"$ & " & $3.6 \mathrm{~cm}$ & $0^{\prime \prime} .18 \times 0^{\prime \prime} .104$ & $5^{\prime \prime} .3$ & $0.023 \mathrm{mJy} \mathrm{bm}^{-1}$ & $"$ \\
\hline " & $"$ & , & $0^{\prime \prime} .26 \times 0.23$ & 5.3 & $0.015 \mathrm{mJy} \mathrm{bm}^{-1}$ & " \\
\hline 2001 Mar 19 & $"$ & 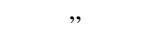 & $0^{\prime \prime} .53 \times 0^{\prime \prime} .53$ & $17^{\prime \prime}$ & $0.04 \mathrm{mJy} \mathrm{bm}^{-1}$ & " \\
\hline
\end{tabular}

Note. ${ }^{a} \Theta_{\text {las }}$ is the angular size of the largest structure to which the observations were sensitive.

\section{OBSERVATIONS}

\subsection{TEXES [Ne II] Data Cube}

NGC 4194 was observed at the NASA Infrared Telescope Facility on Mauna Kea, Hawaii, on the night of 2013 February 2, with the TEXES spectrometer (Lacy et al. 2002). TEXES on the IRTF has a seeing and diffraction-limited beam of $\sim 1$ ". 4 . The observations were taken in the high-resolution mode, with a plate scale $0.36 \times 0.9 \mathrm{~km} \mathrm{~s}^{-1}$ per pixel and spectral resolution $\sim 3 \mathrm{~km} \mathrm{~s}^{-1}$. Ceres was the telluric comparison source and the wavelength scale was set from atmospheric lines. The slit was 29 pixels long and 1".32 wide; it was set north-south and scanned across the galaxy in R.A. steps of $0{ }^{\prime \prime} 7$ to generate data cubes with Nyquist spatial sampling. The total observation time was $2.23 \mathrm{hr}$.

\subsection{Radio Maps}

NGC 4194 was observed at the NRAO Very Large Array $(\mathrm{VLA})^{7}$ in program AN095. A array observations at 6 and $3.6 \mathrm{~cm}$ ( $C$ and $X$ bands) were obtained on 2000 November 17 and B array observations at $X$ band on 2001 March 19. The data were calibrated and reduced with AIPS. Maps were produced with varied weightings and beam sizes; the observing and reduction parameters are given in Table 1.

\subsection{Archival Data \\ 2.3.1. Radio}

We obtained archival data in the radio and infrared regimes from the VLA and from Spitzer. NGC 4194 was observed at $2 \mathrm{~cm}$ ( $U$ band) in the A array for program AE47 and at $6 \mathrm{~cm}$ with the B array in program AS286. Note that the resolution of these B array observations is on the order of the diffractionlimited TEXES beam. The parameters of these maps are given in Table 1.

\subsubsection{Infrared Spectra}

NGC 4194 was observed by Spitzer as part of the IRS Standard Spectra program and the results are in the Spitzer Heritage Archive. It is dominated by the low-excitation lines of [Ne II] and [S III] $18.7 \mu \mathrm{m}$. All the lines appearing in the 9.9-19.6 $\mu \mathrm{m} \mathrm{SH}$ and 18.7-37.2 $\mu \mathrm{m}$ LH modules are in the ratios expected of a starburst galaxy (Sturm et al. 2002); there is no spectral signature of an AGN.

\footnotetext{
7 The National Radio Astronomy Observatory is a facility of the National Science Foundation operated under cooperative agreement by Associated Universities, Inc.
}

\section{H II REGIONS IN THE CENTRAL Kpc OF NGC 4194}

\subsection{Radio Continuum}

Optical and UV observations of NGC 4194 have detected multiple star-forming knots near the nucleus (Weistrop et al. 2004; Hancock et al. 2006), but because of heavy dust obscuration these observations did not penetrate the nucleus itself. Published infrared and radio observations are either of low resolution or at a wavelength not sensitive to the thermal emission of young star-forming regions. The radio observations we report here are the first high-resolution, obscuration-free images of star formation activity in the nucleus of NGC 4194 . We first discuss the spatial distribution and structure of the nucleus, and then the nature of the radio sources.

\subsubsection{Spatial Distribution of Radio Emission}

Maps of NGC 4194 at 6 and $3.6 \mathrm{~cm}$ are shown in Figure 1, and a $2 \mathrm{~cm}$ map, converted from the B1950 to J2000 system, is in Figure 2. This is the first complete and extinctionfree high resolution view of ionized gas structure near the nucleus in NGC 4194. The radio continuum in the central kpc of NGC 4194 has a complex structure which our highest resolution maps barely resolve. The central source ${ }^{8}$ at $\alpha=$ $12^{\mathrm{h}} 14^{\mathrm{m}} 9.68, \delta=+54^{\circ} 31^{\prime} 35^{\prime \prime}$. 8 , appears elongated SE-NW at the lower resolutions. In the highest resolution $3.6 \mathrm{~cm}$ maps it breaks up into 3 sources in a line at p.a. $\approx-40^{\circ}$. Another distinct source lies west of the center. South of the center is a region $\approx 2^{\prime \prime}$ in length, with three distinct sources in a background of extended clumpy emission. These seven distinct sources were fit with single Gaussians and their sizes, positions and fluxes are in Table 2. We discuss the nature of these sources in the next section.

In addition to the compact sources there is extended emission over a $6^{\prime \prime} \times 6^{\prime \prime}$ region. Figure 3 shows a naturally weighted $3.6 \mathrm{~cm}$ image plotted so as to emphasize the lower level emission. It is clearly not axisymmetric. Weak emission connects the central and western radio source with what Beswick et al. (2005) described as an "arm-like feature," and the southern and central source are immersed in what could be a nuclear spiral, a nuclear bar, or part of a star-forming ring or disk. Non-axisymmetric structure has been seen in NGC 4194 and interpreted as a bar (Jog \& Maybhate 2006), but that was at much larger (kpc)

\footnotetext{
8 This source has been identified with the Galactic nucleus in earlier radio maps, and this has been assumed by other authors: Kaaret \& Alonso-Herrero (2008), for example, attribute the X-ray source they find near here to the "nucleus." However kinematic and other considerations, discussed in Section 4.3, cast doubt on that identification, and it will be called "the central source" in this paper.
} 

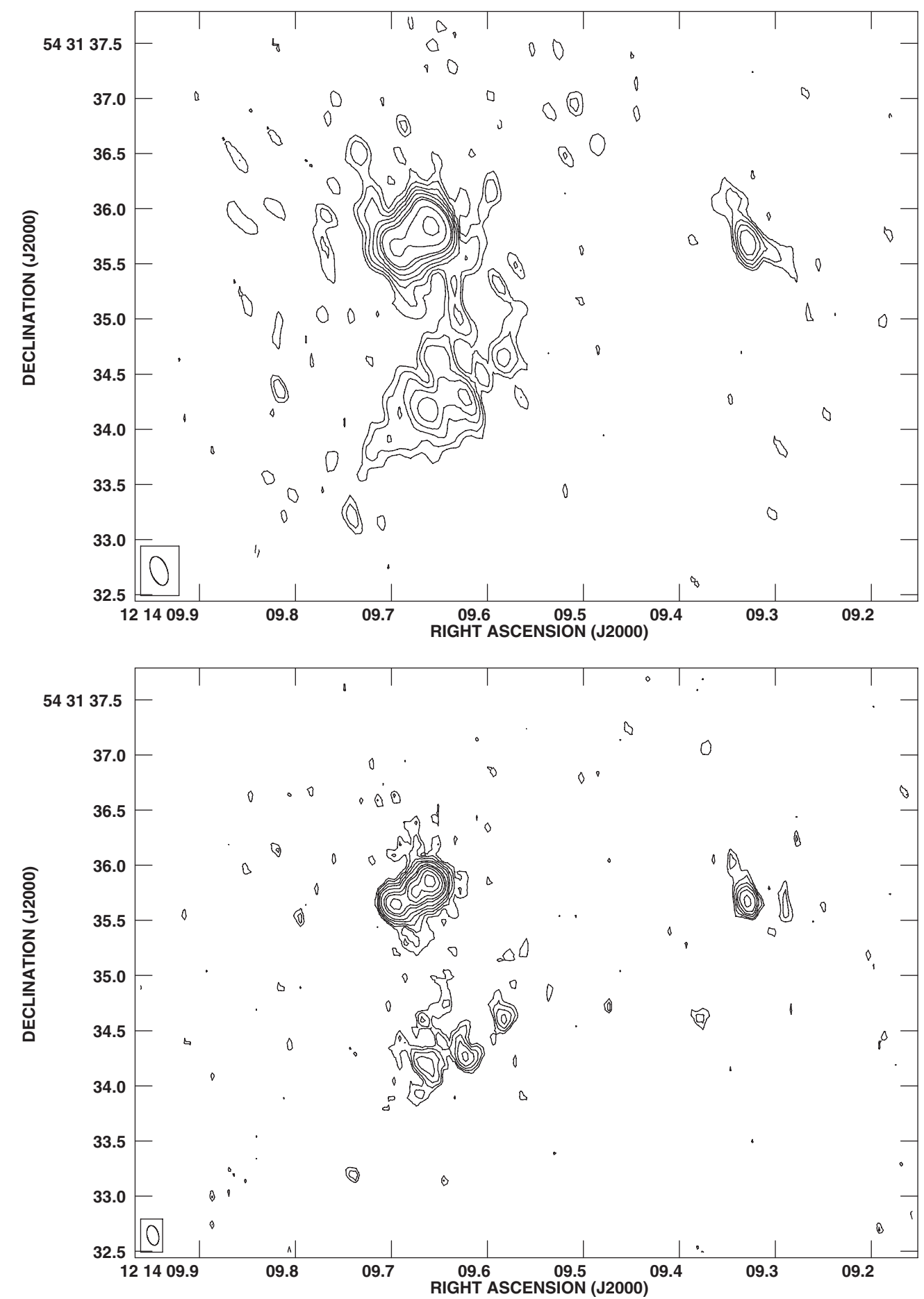

Figure 1. Uniform-weight maps at 6 (top) and $3.6 \mathrm{~cm}$ (bottom). For the $6 \mathrm{~cm}$ map, the B and A array observations were combined to create a data set with a $0.28 \times 0$ "' 19 beam, shown in the lower left, which was sensitive to structures as large $24^{\prime \prime}$. The lowest positive contour is $2.5 \sigma=0.095 \mathrm{mJy}$. The $3.6 \mathrm{~cm}$ map has a $0{ }^{\prime \prime} 18 \times 0{ }^{\prime \prime} 1$ beam, shown, and the lowest positive contour is $2.5 \sigma=0.06 \mathrm{mJy}$. For both maps the levels are spaced logarithmically by factors of $2^{n / 2}$.

radii and does not seem related to the nuclear emission. These structures are discussed in Section 4.

\subsubsection{Thermal Emission and the Spectral Index}

The radio continuum of starburst galaxies combines nonthermal emission from synchrotron sources created in previous generations of stars with thermal free-free emission generated in young $\mathrm{H}$ II regions tracing the current episode of star formation (Condon 1992; Turner \& Ho 1994). Non-thermal processes typically dominate the longest wavelengths $(20 \mathrm{~cm}$ and longer) and thermal free-free the shorter $\mathrm{cm}$ and $\mathrm{mm}$ bands. The radio spectrum can be characterized by the spectral index $\alpha$, such that $S_{v}=v^{\alpha}$ : for pure thermal free-free emission from H II regions, $\alpha=-0.1$, for optically thick emission $\alpha>-0.1$ and can be as great as +2 . For non-thermal synchrotron emission $\alpha$ is steeper, i.e., more negative, than the thermal value. Non-thermal emission of starburst galaxies is observed to have $\alpha$ in the range -0.5 and -1.2 between 21 and $2 \mathrm{~cm}$. The integrated $21 \mathrm{~cm}$ flux of Condon et al. (1990) and the single dish $6 \mathrm{~cm}$ result of 


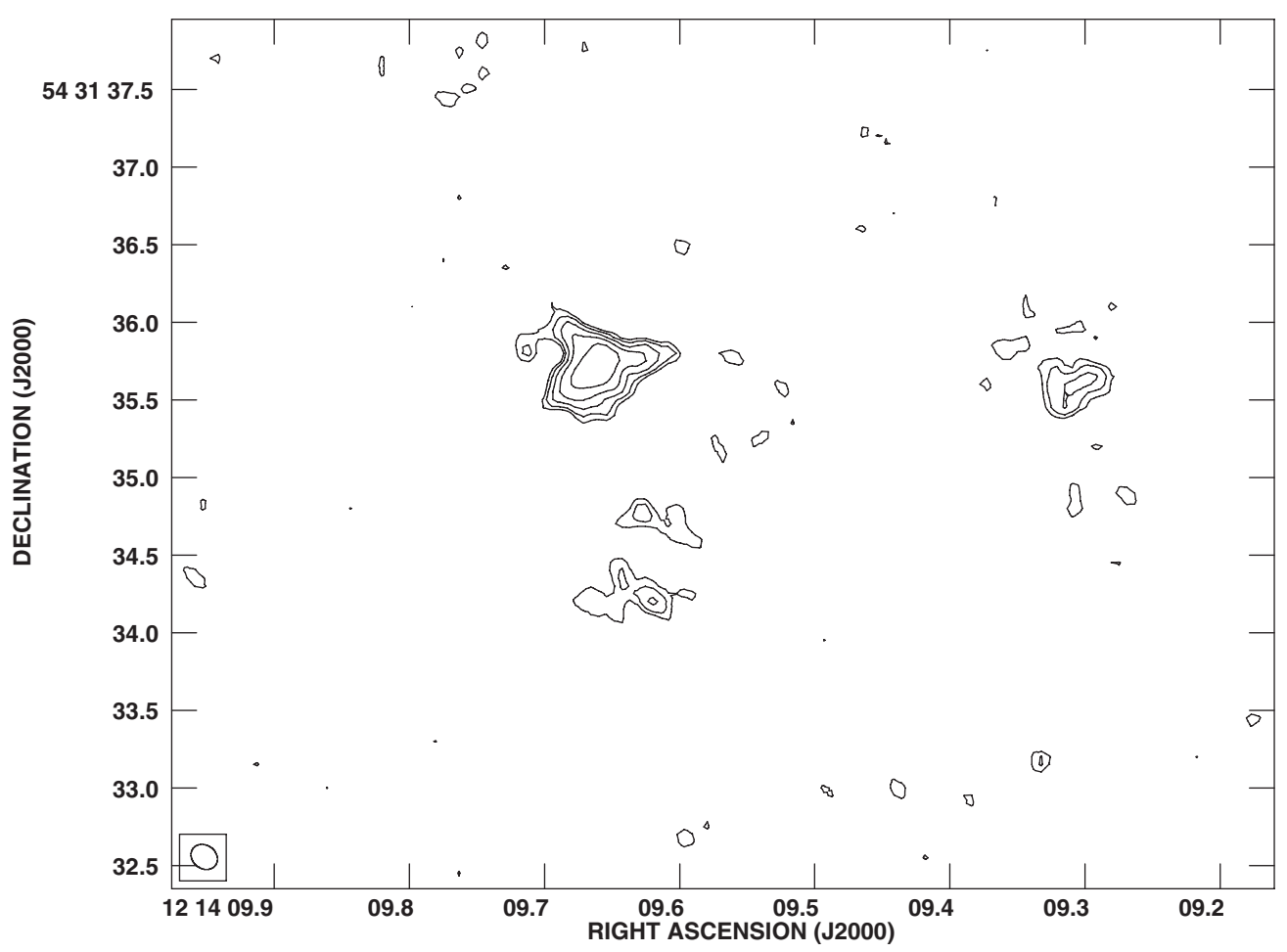

Figure 2. Uniform-weight $2 \mathrm{~cm}$ map. The $0^{\prime \prime} 18 \times 0^{\prime \prime} 15$ beam is shown at lower left, the lowest positive contour is $0.15 \mathrm{mJy}=2.5 \sigma$ and the levels are spaced logarithmically by factors of $2^{n / 2}$.

Table 2

Source Parameters from $3.6 \mathrm{~cm}_{\mathrm{Maps}^{\mathrm{a}}}$

\begin{tabular}{|c|c|c|c|c|c|c|}
\hline ID & R.A. (2000) & Decl. (2000) & Major Axis & Minor Axis & Peak Intensity & Total \\
\hline Central 1 & 121409.68 & 543135.73 & 0 .'46 & $0 ! 22$ & $0.8 \mathrm{mJy} \mathrm{bm}^{-1}$ & $1.2 \mathrm{mJy}$ \\
\hline Central 2 & 121409.67 & 543135.74 & 0.21 & 0.14 & $1.16 \mathrm{mJy} \mathrm{bm}^{-1}$ & $2 \mathrm{mJy}$ \\
\hline Central 3 & 121409.65 & 543135.86 & 0.22 & 0.11 & $1.9 \mathrm{mJy} \mathrm{bm}^{-1}$ & $3.3 \mathrm{mJy}$ \\
\hline W. Source & 121409.326 & 543135.68 & $0 ! 23$ & $0{ }^{\prime} .14$ & $0.46 \mathrm{mJy} \mathrm{bm}^{-1}$ & $0.94 \mathrm{mJy}$ \\
\hline South 1 & 121409.59 & 543134.6 & $0 ! .5$ & 0 .'37 & $0.3 \mathrm{mJy} \mathrm{bm}^{-1}$ & $0.57 \mathrm{mJy}$ \\
\hline South 2 & 121409.63 & 543134.27 & $0^{\prime \prime} 51$ & 0.43 & $0.46 \mathrm{mJy} \mathrm{bm}^{-1}$ & $0.9 \mathrm{mJy}$ \\
\hline South 3 & 121409.66 & 543134.21 & $0^{\prime \prime} 69$ & 0.'513 & $0.46 \mathrm{mJy} \mathrm{bm}^{-1}$ & $1.24 \mathrm{mJy}$ \\
\hline
\end{tabular}

Note. ${ }^{a}$ The nuclear and western cluster fits are from the uniform-weight map with a 0 "' $18 \times 0$ "' 1 beam; the southern clusters are fit from the natural weighted map with a $0.26 \times 0.23$ beam.

Becker et al. (1991) give the spectral index of NGC 4194 as -0.81 between 21 and $6 \mathrm{~cm}$.

The archival $2 \mathrm{~cm}$ observations offer a wider baseline view of the spectral index. We created a $2 \mathrm{~cm}$ image with the same beam size as the $6 \mathrm{~cm}$ and took the spectral index between 6 and $2 \mathrm{~cm}$, which is shown in Figure 4 along with the $2 \mathrm{~cm}$ map contours. The 6-2 cm spectral index shows that the central and southern sources have substantial non-thermal emission and the western is purely thermal. In fact, the spectrum of the western source may actually be rising at $2 \mathrm{~cm}$, consistent with a thermal source of such high emission measure as to be partly optically thick at $6 \mathrm{~cm}$.

The radio continuum at 6 and $3.6 \mathrm{~cm}$ is a mixture of thermal free-free emission and non-thermal synchrotron emission, but the $2 \mathrm{~cm}$ map is mostly thermal. This is shown by comparing the fluxes to those at $2.7 \mathrm{~mm}$, where the non-thermal emission is insignificant and dust emission is negligible. Aalto et al. (2010) measured $4.6 \pm 1 \mathrm{mJy}$ total flux at $2.7 \mathrm{~mm}$ over this entire region. With $\alpha=-0.1$ this predicts total thermal fluxes $5.6 \pm 1 \mathrm{mJy}$ at $2 \mathrm{~cm}$ and $6.3 \pm 1 \mathrm{mJy}$ at $6 \mathrm{~cm}$. So $88 \%$ of the total $2 \mathrm{~cm}$ flux is thermal (but only $15 \%$ of the total $6 \mathrm{~cm}$.) The $2 \mathrm{~cm}$ emission in Figure 2 is thus an excellent high-resolution representation of the extinction-free star formation traced by the free-free emission.

The Lyman continuum rate to maintain this thermal flux is estimated from the prescription of Ho et al. (1990) and the $3 \mathrm{~mm}$ flux to be $N_{\mathrm{Lyc}}=1.0 \times 10^{54}(D / 39 \mathrm{Mpc})^{2} \mathrm{~s}^{-1}$ for the three main star-forming regions of the central $\sim 10^{\prime \prime}$. This corresponds to $L_{\mathrm{OB}}=3 \times 10^{10} L_{\odot}$, and a star formation rate of $\sim 10 M_{\odot} \mathrm{yr}^{-1}$ for a Kroupa 3 Myr starburst based on STARBURST99.

The non-thermal emission in the compact sources of NGC 4194 is probably from recent supernovae. There must have been considerable supernova activity in the recent past: the $3.3 \mathrm{mJy}$ of $3.6 \mathrm{~cm}$ emission which we calculate to be nonthermal requires 450 objects like Cas A. Beswick et al. (2005) found that at $21 \mathrm{~cm}$, where the non-thermal component dominates, the emission appears with a MERLIN beam of 0 ' $17 \times 0$ '. 15 to be a very compact double source. The two components have 12.6 and $5.1 \mathrm{mJy}$ and their positions and separation are consistent with Central Sources 1 and 3 in our 6 and 3.6 maps. There is also a weak $21 \mathrm{~cm}$ source north of the midpoint of 


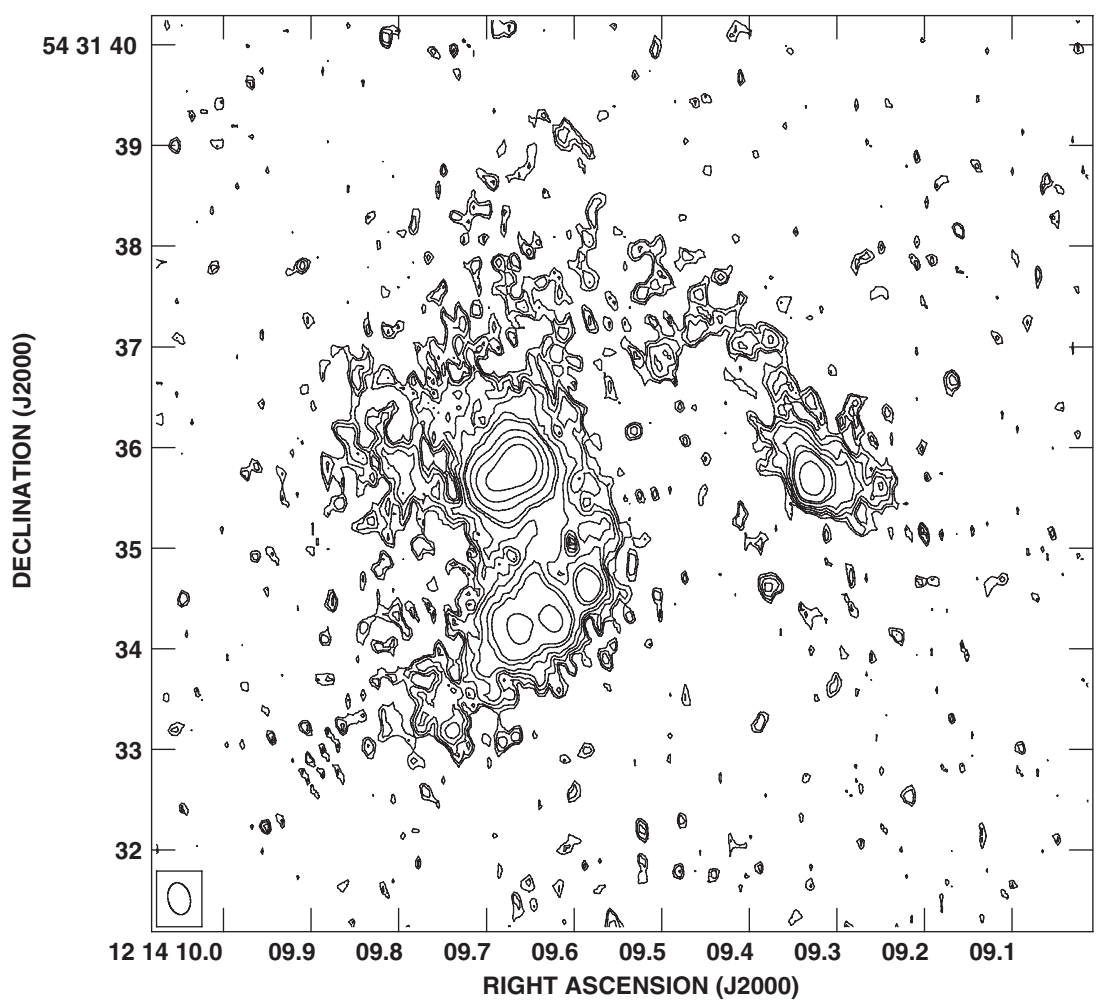

Figure 3. Low-level emission at $3.6 \mathrm{~cm}$. The $0.32 \times 0$ ' 22 beam is shown. The lowest contour is $1.3 \sigma\left(0.02 \mathrm{mJy} \mathrm{bm}^{-1}\right)$ and contour levels are $1,1.4,2,2.8,4,6.25,8$, 16,32 , and 64 .

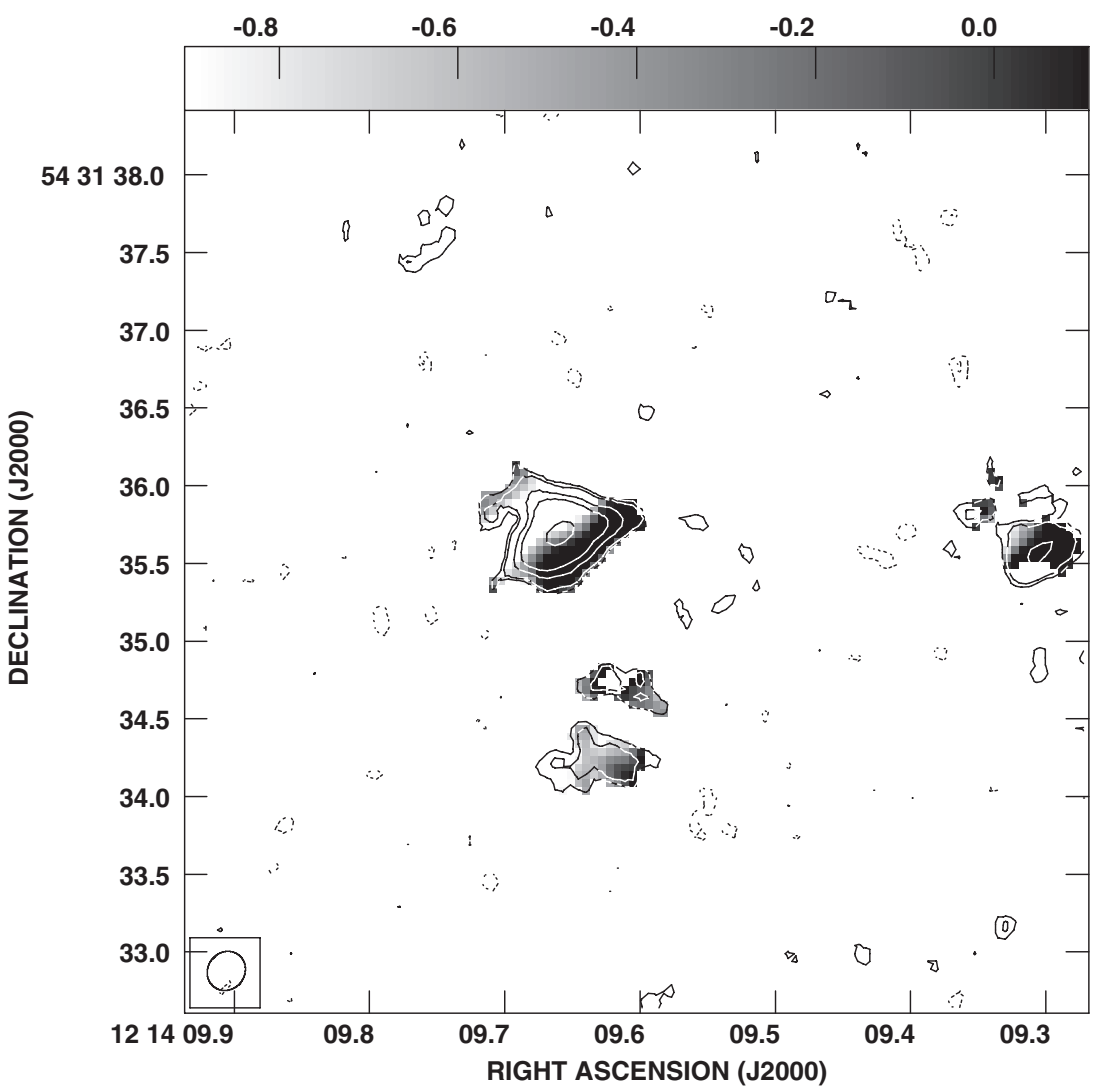

Figure 4. Spectral index between 6 and $2 \mathrm{~cm}$ in grayscale with the $2 \mathrm{~cm}$ intensity in contours. The beam is $0^{\prime \prime} 26 \times 00^{\prime \prime} 23$ and contour interval and levels as in Figure 3 .

the double source with peak flux density $0.7 \mathrm{mJy} \mathrm{bm}^{-1}$; it has no $3.6 \mathrm{~cm}$ counterpart. We cannot, with our present data, map the spectral index with sufficient spatial resolution to locate the non-thermal sources precisely. The $6-2 \mathrm{~cm}$ spectral index hints that the emission is more strongly non-thermal on the northern side of the central source, perhaps related to the weak third source in the $21 \mathrm{~cm}$ map, but that is not conclusive: the $2 \mathrm{~cm}$ data were observed in an epoch different from the $6 \mathrm{~cm}$ and the 


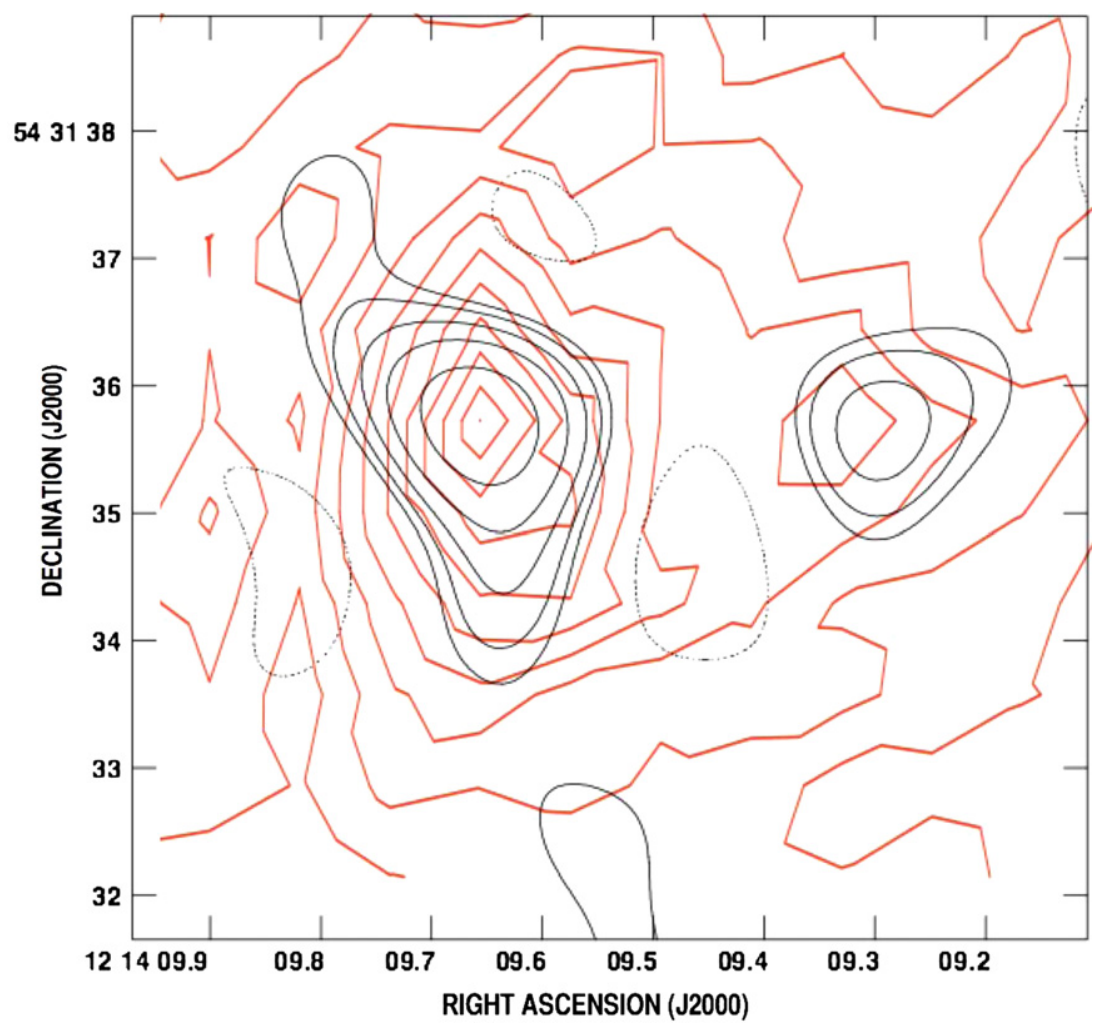

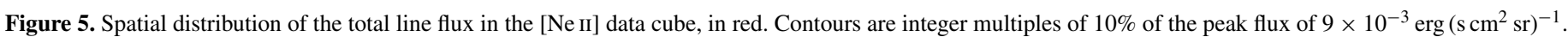
The apparent peak at R.A. 121409.9 is spurious, from a flaw in the detector. Black contours show the $2 \mathrm{~cm}$ radio emission convolved to the [Ne II] beam; contours are integral multiples of $1.68 \mathrm{mJy} \mathrm{bm}^{-1}$ and dashed contours are negative.

(A color version of this figure is available in the online journal.)

precession may not be perfectly accurate. We conclude that thermal and non-thermal emission are mixed in the central and southern sources of NGC 4194 at wavelengths $3.6 \mathrm{~cm}$ and longer.

The purely thermal index of the small western source argues that it is a young $\mathrm{H}$ II region that has not yet produced a significant number of supernovae. From the radio flux we deduce that the Lyman continuum flux in that source is $N_{\text {Lyc }} \approx$ $2.08 \times 10^{53} \gamma^{-1}$, equivalent to $2.08 \times 10^{4} \mathrm{O} 7 \mathrm{~V}$ stars and a star formation rate of $\sim 2 M_{\odot} \mathrm{yr}^{-1}$. If this is one star cluster it is one of the largest super star clusters yet seen; it is much brighter than the central source in NGC 5253 and almost as strong as the SW radio source in NGC 4102 (Beck et al. 2010) and the brightest source in M82 (Tsai et al. 2009). The extended nature of the western source argues that it is probably more than one cluster, however.

In summary, the three main radio sources are intense regions of star formation, but at different evolutionary stages. The central and southern source are old enough that many of their stars have gone supernova. The presence of an X-ray source (presumably an X-ray binary) near the central radio source (Kaaret \& AlonsoHerrero 2008) is another sign of stars in very late stages of evolution. The western source, in contrast, is too young to have formed a significant number of supernovae.

\section{2. [Ne II] Spatial Structure Agrees with the Radio}

The spatial distribution of the [Ne II] emission is found from the data cube by collapsing the cube along the velocity axis. The resulting map is shown in Figure 5, superimposed on a radio map convolved to the same beam size. In the [Ne II] map the western source is a discrete peak, while the southern source is a plateau extending the central source; the radio continuum at the same spatial resolution looks very similar. [Ne II] is a star formation tracer showing the presence of young $\mathrm{H}$ II regions, so its distribution here argues that all the nuclear sources, including those with non-thermal radio spectra, host some recent star formation.

The total flux of [Ne II] summed over the line extent and the region mapped is $1.6 \times 10^{-12} \mathrm{erg} \mathrm{s}^{-1} \mathrm{~cm}^{-2}$, with absolute uncertainty $\approx 30 \%$. The IRS on Spitzer had two pointings of the SH module on this position and measured 4.7 and 5.0 Jy, which is $2 \pm 0.06 \times 10^{-12} \mathrm{erg} \mathrm{s}^{-1} \mathrm{~cm}^{-2}$. There are considerable systemic uncertainties in converting Jy to flux for an unresolved line and in comparing observations of such different resolution as TEXES and IRS; we can say only that the results are consistent with TEXES having recovered the entire Spitzer flux.

\subsection{1. $\left[\mathrm{Ne}^{+}\right]$Abundance}

The strength of the [Ne II] line and of the thermal radio continuum are related because both depend on the emission measure $n_{e}^{2} \ell\left(\mathrm{cm}^{-6} \mathrm{pc}\right)$ If the neon abundance has the solar value of $8.3 \times 10^{-5} \mathrm{n}(\mathrm{H})$ by number, and is all in $\mathrm{Ne}^{+}$(an overestimate, since Spitzer detected [Ne III]), the collision strengths of Osterbrock \& Ferland (2006) give $F([\mathrm{Ne} \mathrm{II}])\left(\mathrm{erg} \mathrm{s}^{-1} \mathrm{~cm}^{-2}\right)=$ $2.0 \times 10^{-10} F_{5 \mathrm{GHz}}(\mathrm{Jy})$. This predicts a total $5 \mathrm{GHz}$ flux of $8 \mathrm{mJy}$ for NGC 4194, compared to the $6 \mathrm{mJy}$ deduced above for thermal radio emission. The sizable uncertainty in our absolute flux calibration permits us to say only that the flux is consistent with a solar or somewhat higher abundance, and even marginally consistent with the slightly less than solar metallicity cited by O'Halloran et al. (2006). 


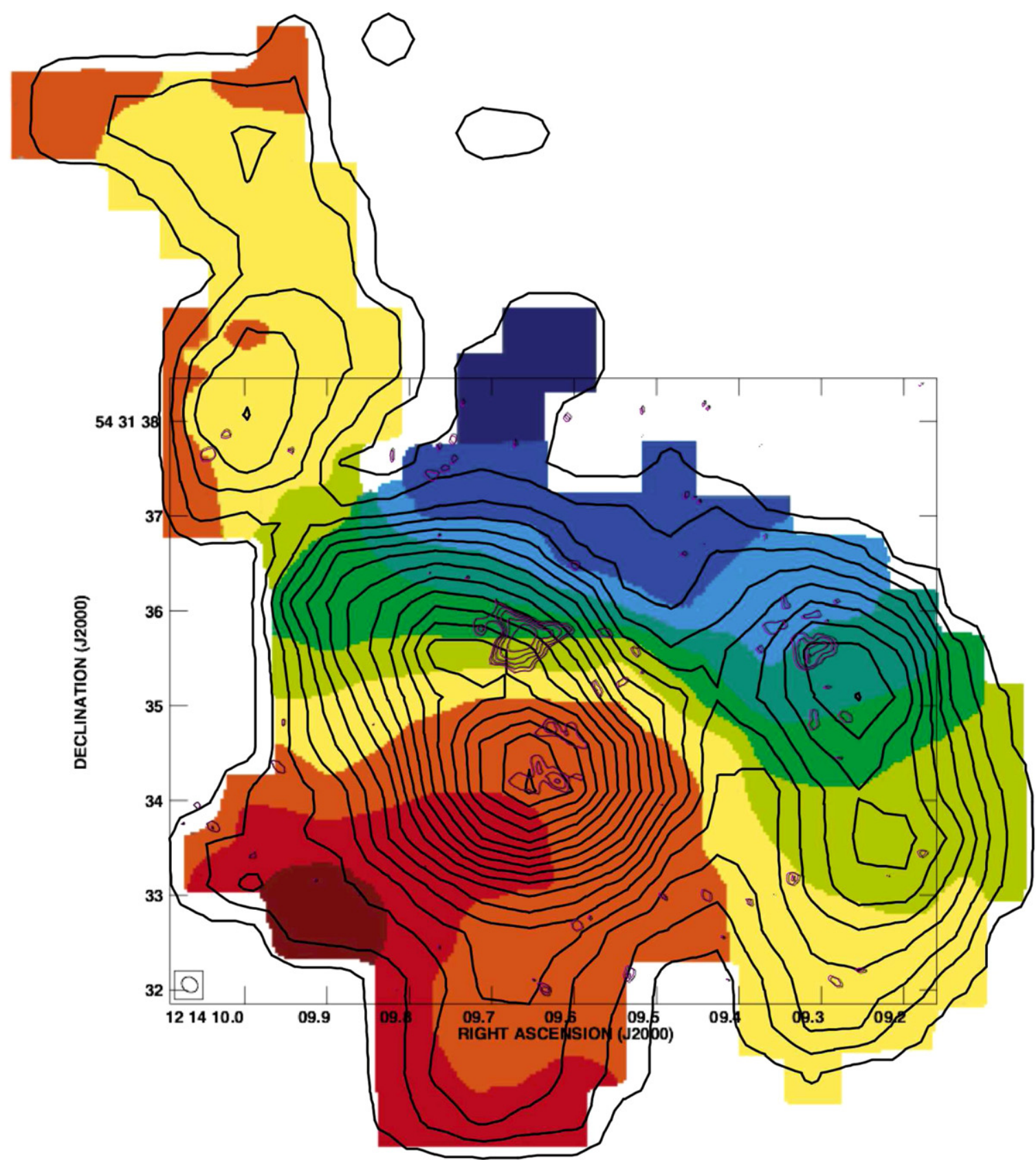

Figure 6. Radio continuum contours of the VLA $2 \mathrm{~cm}$ map are superimposed on the CO integrated intensity and CO velocity field from Aalto \& Huttemeister (2000). The estimated accuracy of the overlay is $\approx 0^{\prime \prime} 2$. The velocity contours are 2400 (blue) to 2640 (red) $\mathrm{km} \mathrm{s}^{-1}$ with $24 \mathrm{~km} \mathrm{~s}^{-1}$ spacing. The CO contours are the total integrated intensity of $\mathrm{CO}$ emission with levels $(0,1.5,3.5, \ldots) \mathrm{Jy} \mathrm{bm}^{-1} \mathrm{~km} \mathrm{~s}^{-1}$. Aalto \& Huttemeister (2000)'s peaks A, B, C, and D go clockwise from the southeast and $\mathrm{E}$ is the northeast feature.

(A color version of this figure is available in the online journal.)

\subsection{Ionized Gas Does Not Coincide with the Molecular Gas}

Aalto \& Huttemeister (2000) used the OVRO interferometer to map the ${ }^{12} \mathrm{CO}(1-0)$ emission in the center of NGC 4194 with beam sizes $1^{\prime \prime} .7-2$.'5 and velocity resolution of $20 \mathrm{~km} \mathrm{~s}^{-1}$. They found that in the region mapped in radio and [ $\mathrm{Ne}$ II] the molecular gas comprises 5 distinct peaks and an extended component. We precessed their figures to the $\mathbf{J} 2000$ system used in the radio continuum observations and show the $2 \mathrm{~cm}$ map overlaid on the CO in Figure 6. Since both the radio and CO maps are interferometer maps, the uncertainty in registration should be negligible. It is clear from the figure that the spatial distribution of molecular gas differs materially from the ionized. First, the brightest radio and [Ne II] source is not perfectly coincident with any of the five $\mathrm{CO}$ peaks, but sits on the northwest side of $\mathrm{CO}$ peak $\mathrm{B}$. The offset is $\approx 0^{\prime} .6$, much larger than the $0{ }^{\prime} 1$ conservatively estimated for the possible error in the precession. Second, the southern clump of radio sources agrees in position with the molecular peak $\mathrm{A}$, but not in relative intensity: the radio and $[\mathrm{Ne}$ II] flux on peak $A$ is much weaker than on peak $\mathrm{B}$, which has the brightest radio emission. The $\mathrm{CO} / 2 \mathrm{~cm}$ ratio on $\mathrm{A}$ is higher than on $\mathrm{B}$ by at least a factor of five. Third, there is no ionized gas detected on peak $\mathrm{D}$ even though it is almost (80\%) as strong in $\mathrm{CO}$ as peak $\mathrm{C}$, which is coincident with the western radio source; the $\mathrm{CO} / 2 \mathrm{~cm}$ ratio in $\mathrm{D}$ must be at least 10 times higher than in $\mathrm{C}$.

We see that the greatest concentration of molecular mass is neither the radio peak nor the geometrical center of the starburst 
activity, and that star formation activity is concentrated on one side of the molecular gas distribution. In other words, the ionized gas does not trace the mass distribution on scales of $\sim 0.5$ or $\sim 80-100 \mathrm{pc}$. The mass of young stars in the Hil regions is not significant on these scales: the total stellar mass in the clusters is less than a few times $10^{7} M_{\odot}$, a small fraction of the total molecular mass of $\approx 2 \times 10^{9} M_{\odot}$ and of the $\approx 10^{8} M_{\odot}$ in each cloud.

This raises a new question: where and what is the Galactic nucleus?. We cannot assume that the nucleus is identical with one of the radio peaks - not even the central source. We return to this in the Conclusions.

\section{KINEMATICS}

The kinematics of molecular gas in NGC 4194 were studied by Aalto \& Huttemeister (2000), who observed CO emission with a $2^{\prime \prime} .0 \times 1^{\prime \prime} .7$ beam and $20 \mathrm{~km} \mathrm{~s}^{-1}$ spectral resolution, and by Aalto et al. (2010) who measured ${ }^{13} \mathrm{CO}$ with a $4^{\prime \prime} .56 \times 33^{\prime \prime} .98$ beam. The atomic gas was observed by Beswick et al. (2005), who had $21 \mathrm{~km} \mathrm{~s}^{-1}$ resolution. The velocity field in NGC 4194 is extremely complex, reflecting the merger that created the galaxy. Aalto \& Huttemeister (2000) and Beswick et al. (2005) agree that in the inner $\approx 0.65 \mathrm{kpc}\left(3^{\prime \prime} .5\right)$ there is a shallow $\mathrm{N}-\mathrm{S}$ velocity gradient of $\approx 60 \mathrm{~km} \mathrm{~s}^{-1} \operatorname{arcsec}^{-1}$, consistent with rotation, that there are significant non-circular motions at larger distances, and that the nuclear region contains some velocity components not due to rotation.

\subsection{Kinematics of Ionized Gas: [Ne II] Distribution}

The TEXES observations are the first to measure ionized gas kinematics in NGC 4194. The spatial distribution of the emission is shown as a two-dimensional grid of spectra in Figure 7(a). The $\left[\mathrm{Ne}\right.$ II] emission in the central $5^{\prime \prime} \times 5^{\prime \prime}$ covers the velocity range $2375-2650 \mathrm{~km} \mathrm{~s}^{-1}$. This is close to the full range over which Beswick et al. (2005) see H I absorption in the central $3^{\prime \prime}$. The overall distribution of the peak velocities also agrees roughly with the H I result: the highest velocities observed are around $2600 \mathrm{~km} \mathrm{~s}^{-1}$ and are in the southern extension, the lowest are around $2400 \mathrm{~km} \mathrm{~s}^{-1}$ and are at the north end of the source, and the northwest is at intermediate velocities of $\sim 2500 \mathrm{~km} \mathrm{~s}^{-1}$.

Besides the similarities there are substantial differences between the ionized gas kinematics, as shown on the spectral grid, and the atomic and molecular findings. While $\mathrm{Ne}^{+}$is the commonest ionization state of neon in a high-metal star formation region like NGC 4194 and is probably widely distributed in the galaxy, the [Ne II] line is collisionally excited and is most readily seen in H II regions of moderate to high density (i.e., $\gtrsim 10^{3} \mathrm{~cm}^{-3}$ ). So the [Ne II] is concentrated in the compact and intense radio sources to which the high-resolution radio maps are sensitive. The spatially isolated $2500 \mathrm{~km} \mathrm{~s}^{-1}$ feature may be straightforwardly associated with the western thermal source. We assign the $2440 \mathrm{~km} \mathrm{~s}^{-1}$ feature to the nuclear source and the $2600 \mathrm{~km} \mathrm{~s}^{-1}$ to the southern emission complex. The spatial resolution is not high enough to separate the central and southern sources completely and they overlap in some positions, creating very complex line profiles (as for example, in Figure 7(b)).

\section{2. [Ne II] Line Profiles}

What can the [Ne II] spectra tell us about the ionized gas motion in this galaxy? The profile of a fine-structure emission line can be formed and influenced by thermal velocity dispersion, gravitational turbulence in a virialized system (such as a bound nebula), and non-turbulent bulk motions. The obvious bulk motion in NGC 4194 is rotation creating a velocity gradient. Does this contribute to the line profiles, or can we treat the sources as kinematically isolated? We explored this question by creating data cubes from the spatial structure of the radio maps and a given velocity gradient. For a reasonable gradient like the $\approx 60 \mathrm{~km} \mathrm{~s}^{-1} \operatorname{arcsec}^{-1}$ of the $\mathrm{CO}$, the gradient does not affect the line widths of the individual sources. The sources are simply too small to include a significant range of the gradient velocities. This justifies analyzing the line profiles in terms of internal, rather than large-scale Galactic, motions.

Thermal velocity dispersion is not an important factor in these line profiles; because of the high mass of neon an $\mathrm{H}$ II region with $T_{e}=7500 \mathrm{~K}$ has FWHM from thermal effects of only $4.1 \mathrm{~km} \mathrm{~s}^{-1}$. The FWHM increases only as the square root of $T_{e}$. Gravitational turbulence in a virialized system will create a Gaussian line whose FWHM can be found from $\sigma=\mathrm{FWHM} / 2.35=(G M / 3 R)^{1 / 2}$ where $R$ is the radius of the system. But since we do not resolve the individual emitting regions in the [Ne II] sources we do not know $R$. Further, the radio maps show that the central and southern [Ne II] source each include several distinct radio sources, presumably super star clusters, whose sum creates the observed lines. So while the gravitational turbulence is probably responsible for most of the width of the line profile, it cannot be satisfactorily calculated from these data for the southern and central sources. For the spatially isolated and apparently simple western source, we show the line summed over a $3 \times 3$ pixel box in Figure 8 along with the best single Gaussian fit. The FWHM is $104 \mathrm{~km} \mathrm{~s}^{-1}$ and the line center is $2480 \mathrm{~km} \mathrm{~s}^{-1} ; \chi^{2} /$ d.o.f. is 1.03 and the residuals do not show structure. The virial velocity relation formally gives $M_{\odot} / R(\mathrm{pc}) \approx 1.4 \times 10^{6} M_{\odot} \mathrm{pc}^{-1}$, while the total stellar mass estimated from the $N_{\text {lyc }}$ and a Kroupa initial mass function (IMF) extending to $0.1 M_{\odot}$ is only $8 \pm 3 \times 10^{5} M_{\odot}$. The source is unresolved with the $0^{\prime \prime} 18(\approx 35 \mathrm{pc})$ beam of the highest resolution radio map. It is likely that even this apparently simple source contains multiple clusters at offset velocities, which make the line appear wider than the true turbulent FWHM. Line profiles in the central and southern sources have FWHM from $85-104 \mathrm{~km} \mathrm{~s}^{-1}$, which formally gives $M / R$ of $0.8-1.4 \times 10^{6} M_{\odot} \mathrm{pc}^{-1}$, but since these sources clearly (from the radio maps) contain multiple sub-sources both the FWHM and the mass concentrations are only upper limits.

\section{3. [Ne II] Kinematics on Large Scales: Position-Velocity Diagrams}

To examine what [Ne II] can tell us about the large-scale kinematics of the nuclear region, we created a data cube binned by 4 spectral pixels and 2 pixels along the slit, to create almost square $0^{\prime \prime} .7 \times 0.7 \times 3.74 \mathrm{~km} \mathrm{~s}^{-1}$ pixels. From this we can see the ionized gas kinematics with minimal spatial averaging. Figure 9(a) shows the line extent as a function of position along a N-S cut through the central source; Figure 9(b) shows an E-W cut, also through the center. The $\mathrm{N}-\mathrm{S}$ position-velocity diagram has an obvious velocity gradient of several tens of $\mathrm{km} \mathrm{s}^{-1} \operatorname{arcsec}^{-1}$ with blue north and red south, very similar to the CO (Aalto \& Huttemeister 2000) and H I (Beswick et al. 2005) results. The ionized gas gradient is $200 \mathrm{~km} \mathrm{~s}^{-1}$ in $4^{\prime \prime} .2$, for a spatial gradient (corrected for the $40^{\circ}$ inclination angle) of $390 \mathrm{~km} \mathrm{~s}^{-1} \mathrm{kpc}^{-1}$. The total velocity extent is similar to the $\mathrm{CO}$ results but the gradient is slightly higher 

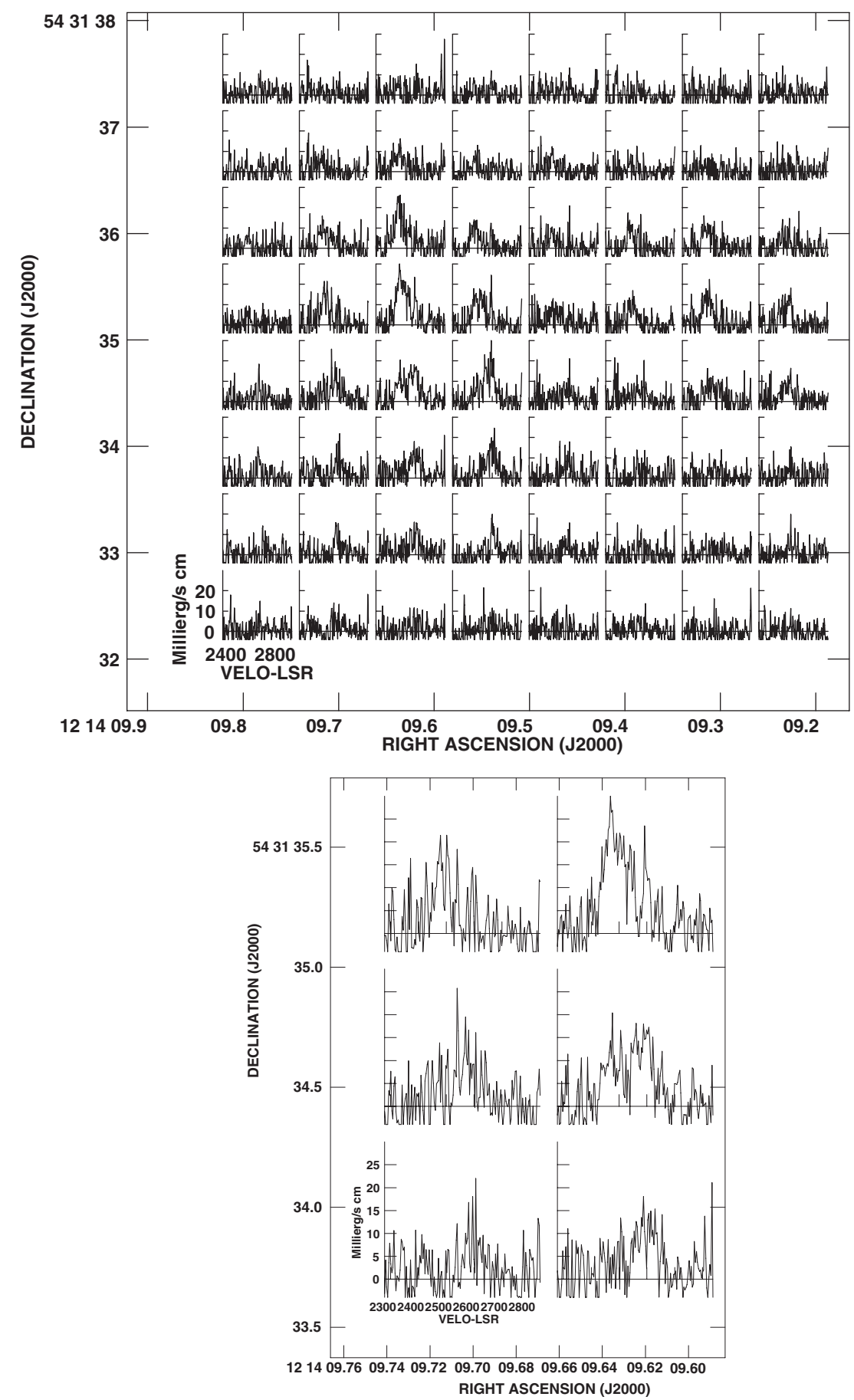

Figure 7. Top: the observed [Ne II] spectra in each pixel. The intensity units are erg $\left(\mathrm{s} \mathrm{cm}^{2} \mathrm{sr}\right)^{-1}$. Bottom: sample pixels from the above figure which are enlarged to show the overlapping velocity components.

than the $320 \mathrm{~km} \mathrm{~s}^{-1} \mathrm{kpc}^{-1}$ of the atomic and molecular gas; this may be because of the very different beam sizes.

The [Ne II] observations average over a very patchy distribution of ionized gas. We checked that this does not distort the position-velocity diagram by comparing it to that of the simulated data cube based on the radio continuum maps; they are consistent. The gradient is not due to averaging over sources at offset velocities: the velocity offsets between the [Ne II] sources result from their positions along an incompletely sampled velocity gradient.

In the E-W position-velocity diagram the central and the western source appear spatially separated and with almost identical velocity ranges of $2400-2550 \mathrm{~km} \mathrm{~s}^{-1}$. There is nothing to suggest an E-W gradient; we find from the simulated cube that if there were a gradient as large as $20 \mathrm{~km} \mathrm{~s}^{-1} \operatorname{arcsec}^{-1}$, it would appear in the position-velocity diagram. There is a separate red feature at $2580 \mathrm{~km} \mathrm{~s}^{-1}$, most clearly in the central source; we believe that to be part of the southern source which was included in the same beam.

\subsection{A Galaxy Core in Solid-body Rotation}

In the previous sections we showed that the none of the $\mathrm{H}$ II regions measured in the [Ne $\mathrm{II}]$ and radio are likely to be a Galactic nucleus. But even if the mass distribution is not 


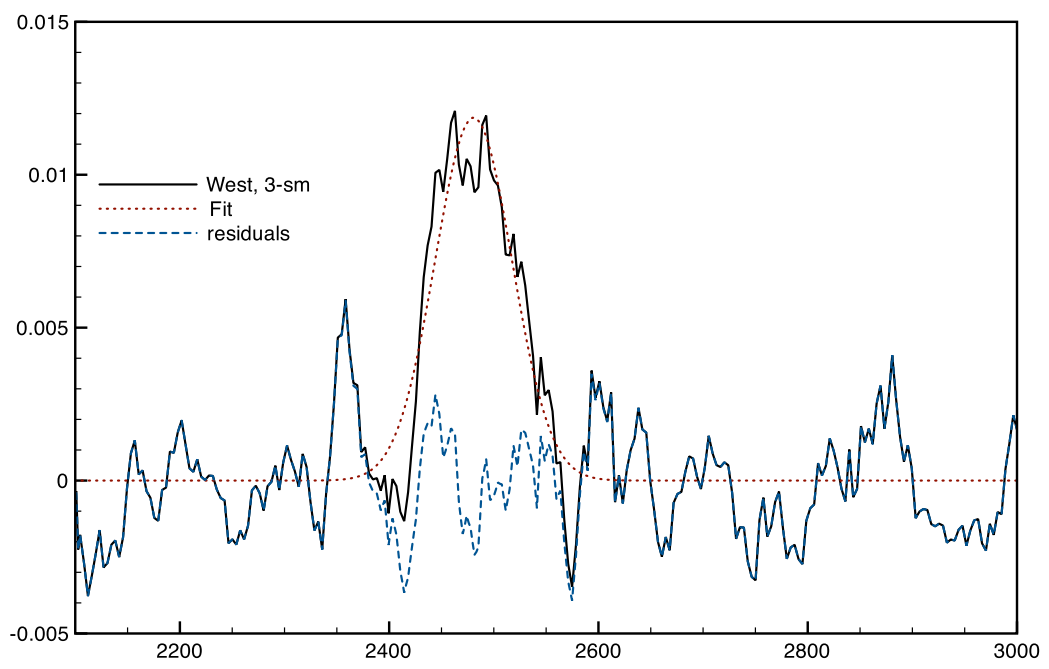

Figure 8. Line profile of the entire western source, with the best single Gaussian fit and the residuals. The fit center is $2480 \mathrm{~km} \mathrm{~s}^{-1}, \mathrm{FWHM} 103.9$, and $\chi^{2} / \mathrm{dof}=1.03$. (A color version of this figure is available in the online journal.)
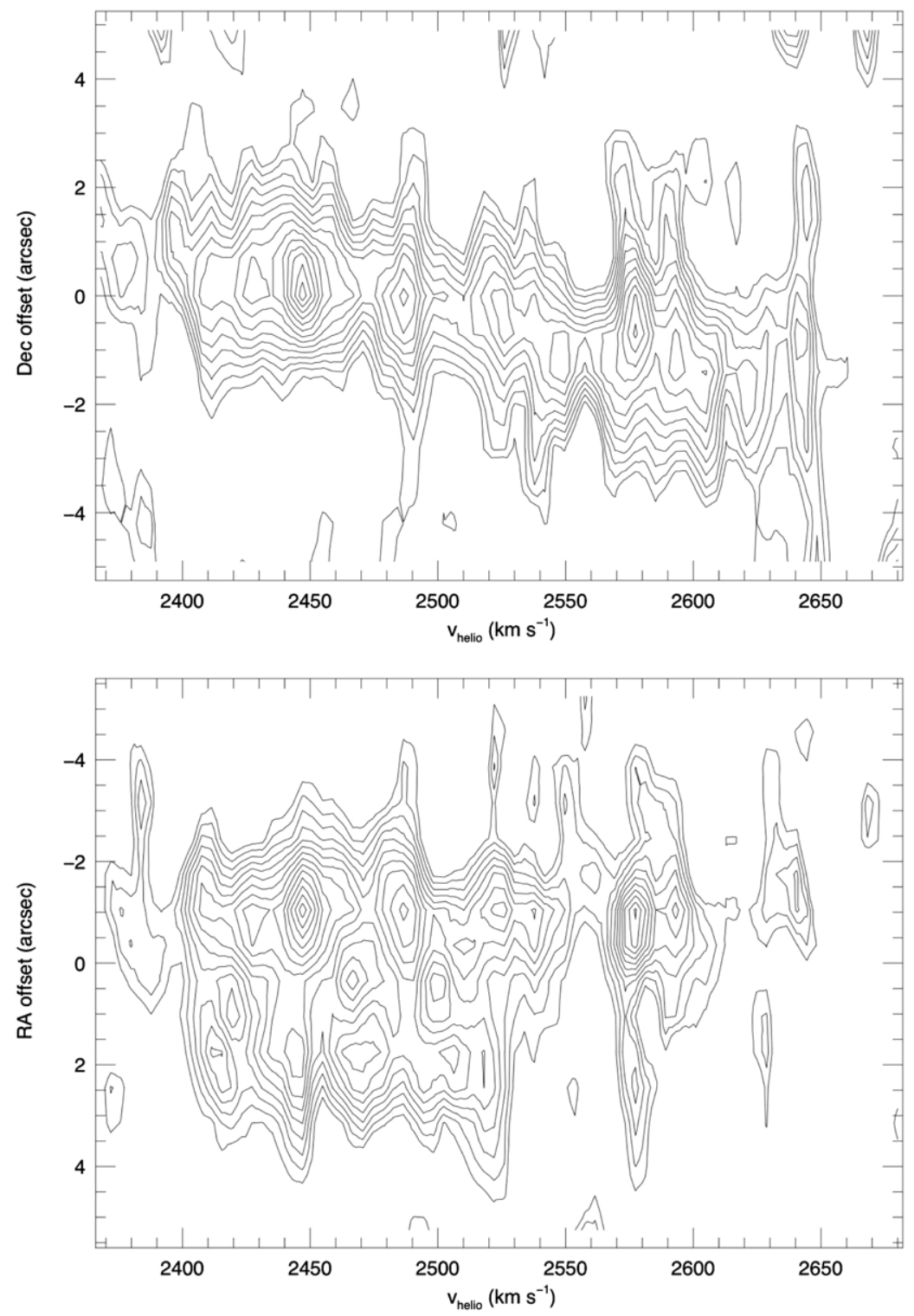

Figure 9. Position-velocity diagrams through the [Ne II] sources. For these diagrams the data was binned by 4 pixels in velocity and by 2 pixels along the slit; the final scale is $0^{\prime \prime} 7 \times 00^{\prime \prime} 7 \times 3.7 \mathrm{~km} \mathrm{~s}^{-1}$. Top is an N-S cut through the nucleus and $\mathrm{N}$ is up; bottom an EW cut through the brightest source with E up; in both, blue is to the left. 
apparent in the $\mathrm{CO}$, radio, and [Ne II] maps, its gravitational effects cannot be hidden. We now find that there is no kinematic sign of a nuclear mass concentration either. In a spiral galaxy there is usually a very broad line on the nucleus and a velocity jump across it; there is no such pattern in NGC 4194. Specifically, the central source, the apparent candidate for the nucleus, has the narrowest line profile of all the [Ne II] sources. The only kinematic hint at a mass concentration is in the $\mathrm{CO}$ velocity field; the isovelocity contours, which are evenly spaced across the main radio and $\mathrm{CO}$ peaks, kink and take on a closer spacing around $\alpha=12^{\mathrm{h}} 14^{\mathrm{m}} 09.5, \delta=+54^{\circ} 31^{\prime} 36^{\prime \prime}$. But that location is between the central and western radio sources and there is no emission peak, at any wavelength, there. Instead, the kinematics are dominated by a smooth gradient $\mathrm{N}-\mathrm{S}$ in both molecular and ionized gas. Smooth gradients imply solidbody rotation. In disk galaxies solid-body rotation is usually associated with rings, and elliptical galaxies, especially those that are post-merger, may host cores several hundred pc in size, kinematically distinct from the rest of the galaxy, with this rotation pattern (de Zeeuw \& Franx 1991).

How can the galaxy have the kinematic signature of solidbody rotation while the ionized gas appears to be a two-armed spiral? If we critically examine the low-level radio emission maps in Figure 3, it is clear that the appearance of "spiral structure" depends very strongly on the southeast elongation of the southern source. The sub-sources fall on a line southeastnorthwest and the emission extends further in a spur in the same direction. The whole southern complex is almost perpendicular to the emission connecting the southern and central sources, so that together with the elongated central source the region suggests a "twin peaks" star formation structure-but again, that contradicts the kinematics, which are not at all like those of the barred spirals where "twin peaks" form. Finally, the "armlike" arc of radio emission connecting the central and western sources lies northwest of the main $\mathrm{CO}$ emission in the region of highest radio-to-CO ratio; it may show a front of star formation proceeding into the clouds, rather than a true spiral arm. So the spiral structure which appears to first glance is less certain on closer inspection.

Let us now work from the opposite direction: how does the ionized gas distribution fit the kinematic results? The central and southern sources are clusters that have formed in a rotating spheroidal core with southern end receding. We cannot rule out that the rotating region is a very thick ring or disk instead of a spheroid; if this is the case the clusters have formed throughout the thickness of the ring. That the western source has almost the same velocity as the central may agree with the finding of Aalto $\&$ Huttemeister (2000) that the velocities of clouds C and D are consistently lower than if they were in solid-body rotation with $\mathrm{A}$ and $\mathrm{B}$. The western source, like its host cloud, may not have yet settled into equilibrium.

\section{CONCLUSIONS}

We have presented high-resolution radio continuum maps at $6,3.6$, and $2 \mathrm{~cm}$, and a high spectral-resolution velocity-position cube in the [Ne II] $12.8 \mu \mathrm{m}$ emission line, of the central $1.3 \mathrm{kpc}$ of NGC 4194. These maps are the first high-resolution, extinction-free, measurements of ionized gas in the center of this galaxy and trace the star formation regions with resolution up to $0^{\prime \prime} 18(\sim 35 \mathrm{pc})$. We find that:

1. NGC 4194 hosts a nuclear starburst that has formed multiple compact sources, apparently groups of embedded super star clusters, within a $3^{\prime \prime}$ radius. The low-level radio emission suggests a "nuclear spiral" but that is not confirmed by the kinematics.

2. Based on the thermal radio emission at $2 \mathrm{~cm}$, which agrees well with previous observations at $2.7 \mathrm{~mm}$, we find for the central $\sim 10^{\prime \prime}$ region a thermal free-free flux of $S_{2 \mathrm{~cm}}^{\text {thermal }}=5.6 \pm 1 \mathrm{mJy}$, which implies a Lyman continuum rate of $N_{\mathrm{Lyc}}=1.0 \times 10^{54}(D / 39 \mathrm{Mpc})^{2} \mathrm{~s}^{-1}$. For a Kroupa IMF and a 3 Myr starburst, this implies a star formation rate of $\sim 10 M_{\odot} \mathrm{yr}^{-1}$, and a luminosity in massive young stars of $L_{\mathrm{OB}}=3 \times 10^{10} L_{\odot}$, about a third of the total infrared luminosity of $L_{\mathrm{IR}}=8.5 \times 10^{10} L_{\odot}$. The star formation in this galaxy is dispersed widely over the inner $10^{\prime \prime}(1.9 \mathrm{kpc})$ region. Our radio observations are not sensitive to regions with $2 \mathrm{~cm}$ fluxes less than $\sim 0.1 \mathrm{mJy}$, or $N_{\text {Lyc }}<2 \times 10^{52}(D / 39 \mathrm{Mpc})^{2} \mathrm{~s}^{-1}$, so it is possible that the remaining infrared luminosity arises in smaller, and widely dispersed, star-forming regions within the system.

3. Of the compact emission sources that are bright enough to analyze, one has a pure thermal or slightly rising spectrum, typical of a very young embedded H II region. The others are older and have a mix of thermal and significant nonthermal emission. The [Ne II] line velocity dispersions are consistent with the gravitational effects of many dense star clusters.

4. The ionized gas has a smooth $\mathrm{N}-\mathrm{S}$ velocity gradient of $390 \mathrm{~km} \mathrm{~s}^{-1} \mathrm{kpc}^{-1}$ across the observed region, consistent with a core in solid-body rotation.

5. None of the radio sources can be convincingly identified with a Galactic nucleus. The nuclei of the original merger partners have not been identified.

6. The $\mathrm{CO}$ velocity field shows that a mass concentration is present at $\alpha=12^{\mathrm{h}} 14^{\mathrm{m}} 09^{\mathrm{s}} .5, \delta=+54^{\circ} 31^{\prime} 36^{\prime \prime}$. This location is in between and roughly equidistant from the three star formation regions, and there is no star formation detected there.

How can the last two items on this list be reconciled with the scenario that the Medusa is a minor merger? Each of the merger partners presumably hosted a nucleus of mass $10^{6}-10^{8} M_{\odot}$ that would be detected via their influence on the kinematics. It is possible, and would be consistent with the simulations, to have both original nuclei now included in the mass concentration detected in the isovelocity contours. But in this case the nuclei must be devoid of star formation and ionized gas, or they would be detected in the radio and [Ne II]. The gas and stars in the center of NGC 4194 have not come to equilibrium and the starburst may continue in regions now quiescent. Measurements of ionised and molecular gas with higher spatial resolution, and simulations exploring the central kpc, could perhaps find more clues to the behavior of this remarkable galaxy.

TEXES observations at the IRTF were supported by NSF AST-0607312 and by AST-0708074 to Matt Richter. This research has made use of the NASA and IPAC Extragalactic Database (NED) which is operated by the Jet Propulsion Laboratory, Caltech, under contract with NASA. We thank an anonymous referee for careful and thoughtful comments.

\section{REFERENCES}

Aalto, S., Beswick, R., \& Jutte, E. 2010, A\&A, 552, A59

Aalto, S., \& Huttemeister, S. 2000, A\&A, 362, 42

Achtermann, J. M., \& Lacy, J. H. 1995, ApJ, 439, 163 
Beck, S. C., Lacy, J., \& Turner, J. 2010, ApJ, 722, 1175

Beck, S. C., Lacy, J., Turner, J., et al. 2012, ApJ, 755, 59

Beck, S. C., Turner, J., Lacy, J., Greathouse, T., \& Lahad, O. 2013, ApJ, 767,53

Becker, R. H., White, R. L., \& Edwards, A. L. 1991, ApJS, 75, 1

Beswick, R. J., Aalto, S., Pedlar, A., \& Huttemeister, S. 2005, A\&A, 44, 791

Condon, J. 1992, ARA\&A, 30, 575

Condon, J. J., Helou, G., Sanders, D. B., \& Soifer, B. T. 1990, ApJS, 73, 359

de Zeeuw, T., \& Franx, M. 1991, ARA\&A, 29, 239

Hancock, M., Weistrop, D., Nelson, C. H., \& Kaiser, M. E. 2006, AJ, 131, 135

Hattori, T., Yoshida, M., Ohtani, H., et al. 2004, AJ, 127, 736

Ho, P., Beck, S. C., \& Turner, J. 1990, ApJ, 349, 57

Jog, C., \& Maybhate, A. 2006, MNRAS, 370, 891

Kaaret, P., \& Alonso-Herrero, A. 2008, ApJ, 682, 1020

Kartaltepe, J. S., Sanders, D. B., Le Floc'h, E., et al. 2010, ApJ, 721, 98
Lacy, J., Richter, M., Greathouse, T., \& Zhu, Q.-F. 2002, PASP, 114, 153

Manthey, E., Aalto, S., Httemeister, S., \& Oosterloo, T. A. 2008, A\&A, 484, 693

O’Halloran, B., Satyapal, S., \& Dudik, R. P. 2006, ApJ, 641, 795

Osterbrock, D., \& Ferland, G. 2006, Astrophysics of Gaseous Nebulae and Active Galactic Nuclei (Sausalito, CA: Univ. Science Books)

Sanders, D. B., Soifer, B. T., Elias, J. H., Neugebauer, G., \& Matthews, K. 1988, ApJL, 328, L35

Sturm, E., Lutz, D., Verma, A., et al. 2002, A\&A, 393, 821

Tsai, C.-W., Turner, J. L., Beck, S. C., Meier, D. S., \& Ho, P. T. P. 2009, AJ, 137,4655

Turner, J. L., \& Ho, P. T. H. 1994, ApJ, 421, 122

Weistrop, D., Eggers, D., Hancock, M., et al. 2004, AJ, 127, 1360

Zhu, Q-F., Lacy, J. H., Jaffe, D., Richter, M., \& Greathouse, T. 2008, ApJS, 177,584 